\title{
SOFTENING THE COMPLEXITY OF ENTROPIC MOTION ON CURVED STATISTICAL MANIFOLDS
}

\author{
Carlo Cafaro ${ }^{1}$, Adom Giffin ${ }^{2}$, Cosmo Lupo $^{3}$, Stefano Mancini ${ }^{4}$ \\ 1, 3, ${ }^{4}$ School of Science and Technology, Physics Division, \\ University of Camerino, I-62032 Camerino, Italy and \\ ${ }^{2}$ Princeton Institute for the Science and Technology of Materials, \\ Princeton University, Princeton, NJ 08540, USA
}

\begin{abstract}
We study the information geometry and the entropic dynamics of a $3 D$ Gaussian statistical model. We then compare our analysis to that of a $2 D$ Gaussian statistical model obtained from the higher-dimensional model via introduction of an additional information constraint that resembles the quantum mechanical canonical minimum uncertainty relation. We show that the chaoticity (temporal complexity) of the $2 D$ Gaussian statistical model, quantified by means of the Information Geometric Entropy (IGE) and the Jacobi vector field intensity, is softened with respect to the chaoticity of the $3 D$ Gaussian statistical model.
\end{abstract}

PACS numbers: Probability Theory (02.50.Cw), Riemannian Geometry (02.40.Ky), Chaos (05.45.-a), Complexity (89.70.Eg), Entropy (89.70.Cf).

\section{INTRODUCTION}

A very important problem in modern science concerns the description and the understanding of the elusive concept of complexity [1 3]. There are pragmatic reasons of primary importance in quantum information science that justify the study of complexity, for example the problem of quantifying how complex is quantum motion [4]. Unfortunately, our knowledge of the basic connections between complexity, dynamical stability, and chaoticity in a fully quantum domain is not satisfactory [4, 5]. The concept of complexity is very difficult to define, its origin is not fully understood [6 11] and it is mainly for this reason that several quantitative measures of complexity have appeared in the scientific literature [1 3] . In classical physics, complexity measures are settled in a much better way. The Kolmogorov-Sinai metric entropy [12], that is the sum of all positive Lyapunov exponents [13], is a powerful indicator of unpredictability in classical systems and it measures the algorithmic complexity of classical trajectories [14 17]. Other known measures of complexity are the logical depth [18], the thermodynamic depth [19], the computational complexity [20], the stochastic complexity [21] and many more. Ideally, a good definition of complexity should be mathematically rigorous and intuitive at the same time so that we are able to tackle complexity-related problems in computation theory and statistical physics as well. Of course, a quantitative measure of complexity is truly useful if its range of applicability is not limited to few unrealistic applications. For this reason, in order to properly define complexity measures, the purpose for defining such a measure and what it is intended to capture should be clearly stated.

Another problem of great theoretical interest is understanding how to compare quantum and classical chaos (temporal complexity) and explaining the reason why the former is weaker than the latter 22 24]. Indeed, it is commonly conjectured that the weakness of quantum chaos may be a consequence of the Heisenberg uncertainty relation [22 24]. It is also known that a quantum description of chaos is qualitatively different from a classical description and that the later cannot simply be considered an approximation of the former. Indeed, the only trace of quantum theory which a classical description may retain is the canonical Heisenberg's uncertainty relation, namely a minimum spread of order $\hbar^{n}$ in the $2 n$-dimensional phase space [25] ( $\hbar \stackrel{\text { def }}{=} \frac{h}{2 \pi}$ where $h$ is Planck's constant).

Motivated by such considerations and following the line of reasoning presented in [26], we will discuss in this paper the manner in which the degree of complexity changes for a statistical model (the probabilistic description of a physical system) in the presence of incomplete knowledge ("softening effects") when the entropic dynamics (or, information-constrained dynamics) [27] on the underlying curved statistical manifolds becomes more constrained. By "softening effects" we mean any attenuation in the asymptotic temporal growth of complexity indicators of motion. Our aim is to reduce the probabilistic description of dynamical systems in the presence of partial knowledge to information geometry (Riemannian geometry applied to probability theory, see 28]) and inductive inference [29 33]. To achieve such a task, we have developed a theoretical framework termed IGAC, Information Geometric Approach to Chaos [34, 35], where information geometric techniques are combined with Maximum relative Entropy methods 29 33] to study the complexity of informational geodesic flows on curved statistical manifolds (statistical models) underlying the probabilistic description of physical systems in the presence of incomplete information. IGAC is the information geometric analogue of conventional geometrodynamical approaches to chaos [36, 37] where the classical configuration space is being replaced by a statistical manifold with the additional possibility of considering chaotic dynamics arising from non conformally flat metrics (the Jacobi metric is always conformally flat, instead). It is 
an information-geometric extension of the Jacobi geometrodynamics (the geometrization of a Hamiltonian system by transforming it to a geodesic flow [38]). The reformulation of dynamics in terms of a geodesic problem allows the application of a wide range of well-known geometrical techniques in the investigation of the solution space and properties of the equation of motion. The power of the Jacobi reformulation is that all of the dynamical information is collected into a single geometric object in which all the available manifest symmetries are retained- the manifold on which geodesic flow is induced. For example, integrability of the system is connected with existence of Killing vectors and tensors on this manifold. The sensitive dependence of trajectories on initial conditions, which is a key ingredient of chaos, can be investigated from the equation of geodesic deviation (the so-called Jacobi-Levi-Civita equation or JLC equation). Within the IGAC formalism, conventional signatures of the chaoticity of classical dynamics emerge via the asymptotic exponential divergence of the JLC vector field intensity and/or via the specific behavior of the asymptotic growth of the Information Geometric Entropy (IGE) (i.e., the logarithm geodesic volume) [26].

In view of the above-mentioned considerations, we discuss here the information geometry and the informationconstrained dynamics of a $3 D$ Gaussian statistical model. We then compare our analysis to that of a $2 D$ Gaussian statistical model obtained from the higher-dimensional model via introduction of an additional information constraint that resembles the quantum mechanical canonical minimum uncertainty relation. We show that the chaoticity (temporal complexity) of the $2 D$ Gaussian statistical model, quantified by means of the IGE [26] and the Jacobi vector field intensity, is softened (that is, attenuated) with respect to the chaoticity of the $3 D$ Gaussian statistical model. In view of the similarity between the information constraint on the variances and the phase-space coarse-graining imposed by the Heisenberg uncertainty relations, we suggest that our work provides a possible way of explaining the phenomenon of suppression of classical chaos operated by quantization.

The layout of this article is as follows. In Section II, we present the basic differential geometric properties of both the $3 D$ and $2 D$ Gaussian statistical models. In Section III, we describe the geodesic paths on the curved statistical manifolds underlying the entropic dynamics of the three and two-dimensional statistical models. In Section IV, we study the chaotic properties of the information-constrained dynamics on the underlying curved statistical manifolds by means of the IGE and the Jacobi vector field intensity. Our final remarks appear in Section V.

\section{GEOMETRY OF THE STATISTICAL MODELS}

The statistical models studied are a $3 D$ uncorrelated Gaussian model and a $2 D$ Gaussian statistical model obtained from the higher-dimensional model via introduction of an additional information constraint that resembles the canonical minimum uncertainty relation in quantum theory. For a brief and recent overview on the IGAC, we refer to [26]. Note that the dimensionality $(2 D, 3 D)$ pertains to the macroscopic variables.

\section{A. The 3D Statistical Model}

The probability distributions $p\left(x, y \mid \mu_{x}, \sigma_{x}, \sigma_{y}\right)$ that characterize the $3 D$ Gaussian statistical model are given by,

$$
p\left(x, y \mid \mu_{x}, \sigma_{x}, \sigma_{y}\right) \stackrel{\text { def }}{=} \frac{1}{2 \pi \sigma_{x} \sigma_{y}} \exp \left[-\frac{1}{2 \sigma_{x}^{2}}\left(x-\mu_{x}\right)^{2}-\frac{1}{2 \sigma_{y}^{2}} y^{2}\right],
$$

with $\sigma_{x}$ and $\sigma_{y}$ in $\mathbb{R}_{0}^{+}$and $\mu_{x}$ in $\mathbb{R}$. The Gaussian here is two dimensional in its microscopic space $(x, y)$ but three dimensional in its macroscopic (contextual or conditionally given parameters) space. We will now relax this conditionality to explore the space of Gaussians described by $\mu_{x}, \sigma_{x}$ and $\sigma_{y}$. The infinitesimal Fisher-Rao line element $d s_{3 D}^{2}$ reads,

$$
d s_{3 D}^{2}=\sum_{l, m=1}^{3} g_{l m}^{(3 D)}(\theta) d \theta^{l} d \theta^{m}=\frac{1}{\sigma_{x}^{2}} d \mu_{x}^{2}+\frac{2}{\sigma_{x}^{2}} d \sigma_{x}^{2}+\frac{2}{\sigma_{y}^{2}} d \sigma_{y}^{2},
$$

where the Fisher-Rao information metric $g_{l m}(\theta)$ is defined as [28],

$$
g_{l m}^{(3 D)}(\theta) \stackrel{\text { def }}{=} \int d x d y p\left(x, y \mid \mu_{x}, \sigma_{x}, \sigma_{y}\right) \frac{\partial \log p\left(x, y \mid \mu_{x}, \sigma_{x}, \sigma_{y}\right)}{\partial \theta^{l}} \frac{\partial \log p\left(x, y \mid \mu_{x}, \sigma_{x}, \sigma_{y}\right)}{\partial \theta^{l}},
$$

with $\theta \equiv\left(\theta^{1}, \theta^{2}, \theta^{3}\right) \stackrel{\text { def }}{=}\left(\mu_{x}, \sigma_{x}, \sigma_{y}\right)$ and where $g_{l m}^{(3 D)}(\theta)$ has the following matrix representation,

$$
g_{l m}^{(3 D)}(\theta)=\left(\begin{array}{ccc}
\frac{1}{\sigma_{x}^{2}} & 0 & 0 \\
0 & \frac{2}{\sigma_{x}^{2}} & 0 \\
0 & 0 & \frac{2}{\sigma_{y}^{2}}
\end{array}\right)
$$


Using (4), it follows that the non-vanishing Christoffel connection coefficients $\Gamma_{i j}^{k}$ [39],

$$
\Gamma_{i j}^{k}=\frac{1}{2} g^{k m}\left(\partial_{i} g_{m j}+\partial_{j} g_{i m}-\partial_{m} g_{i j}\right)
$$

are given by,

$$
\Gamma_{12}^{1}=\Gamma_{21}^{1}=-\frac{1}{\sigma_{x}}, \Gamma_{11}^{2}=\frac{1}{2 \sigma_{x}}, \Gamma_{22}^{2}=-\frac{1}{\sigma_{x}}, \Gamma_{33}^{3}=-\frac{1}{\sigma_{y}}
$$

The scalar curvature $\mathcal{R}^{(3 D)}$ of the statistical manifold of probability distributions in (1) is given by,

$$
\mathcal{R}^{(3 D)}=g^{11}(\theta) R_{11}+g^{22}(\theta) R_{22}+g^{33}(\theta) R_{33},
$$

with $g^{l m} g_{m k}=\delta_{k}^{l}$ and the Ricci curvature tensor $R_{i j}$ defined as [39],

$$
R_{i j} \stackrel{\text { def }}{=} \partial_{k} \Gamma_{i j}^{k}-\partial_{j} \Gamma_{i k}^{k}+\Gamma_{i j}^{k} \Gamma_{k n}^{n}-\Gamma_{i k}^{m} \Gamma_{j m}^{k}
$$

Substituting (6) into (8), it turns out that the non vanishing components of $R_{i j}$ are,

$$
R_{11}=-\frac{1}{2 \sigma_{x}^{2}}, R_{22}=-\frac{1}{\sigma_{x}^{2}}
$$

Thus, the scalar curvature becomes $\mathcal{R}^{(3 D)}=-1$. We finally point out the only non-vanishing component of the Riemann curvature tensor $R_{\mu \nu \rho}^{\alpha}$ [39],

$$
R_{\mu \nu \rho}^{\alpha} \stackrel{\text { def }}{=} \partial_{\nu} \Gamma_{\mu \rho}^{\alpha}-\partial_{\rho} \Gamma_{\mu \nu}^{\alpha}+\Gamma_{\beta \nu}^{\alpha} \Gamma_{\mu \rho}^{\beta}-\Gamma_{\beta \rho}^{\alpha} \Gamma_{\mu \nu}^{\beta}
$$

is given by,

$$
R_{212}^{1}=-\frac{1}{\sigma_{x}^{2}}
$$

As a final remark, note that

$$
\mathcal{R}^{(3 D)} \equiv\left(R_{1212}+R_{2121}\right) g^{11}(\theta) g^{22}(\theta)=-1
$$

in agreement with Eq. (77).

\section{B. The 2D Statistical Model}

The probability distributions $p\left(x, y ; \mu_{x}, \sigma\right)$ that characterize the $2 D$ Gaussian statistical model are given by,

$$
p\left(x, y ; \mu_{x}, \sigma\right) \stackrel{\text { def }}{=} \frac{1}{2 \pi \Sigma^{2}} \exp \left[-\frac{1}{2 \sigma^{2}}\left(x-\mu_{x}\right)^{2}-\frac{\sigma^{2}}{2 \Sigma^{4}} y^{2}\right],
$$

with $\sigma$ in $\mathbb{R}_{0}^{+}$and $\mu_{x}$ in $\mathbb{R}$. The probability distribution $p\left(x, y ; \mu_{x}, \sigma\right)$ may be obtained from $p\left(x, y \mid \mu_{x}, \sigma_{x}, \sigma_{y}\right)$ with the addition of the following macroscopic constraint,

$$
\sigma_{x} \sigma_{y}=\Sigma^{2}
$$

where $\Sigma^{2}$ is a constant belonging to $\mathbb{R}_{0}^{+}$and $\sigma_{x} \equiv \sigma$. The macroscopic constraint (14) resembles the quantum mechanical canonical minimum uncertainty relation where $x$ denotes the position of a particle and $y$ its conjugate momentum. The infinitesimal Fisher-Rao line element $d s_{2 D}^{2}$ reads,

$$
d s_{2 D}^{2}=\sum_{l, m=1}^{2} g_{l m}^{(2 D)}(\theta) d \theta^{l} d \theta^{m}=\frac{1}{\sigma^{2}} d \mu_{x}^{2}+\frac{4}{\sigma^{2}} d \sigma^{2}
$$


where the Fisher-Rao information metric $g_{l m}(\theta)$ is defined as,

$$
g_{l m}^{(2 D)}(\theta) \stackrel{\text { def }}{=} \int d x d y p\left(x, y ; \mu_{x}, \sigma\right) \frac{\partial \log p\left(x, y ; \mu_{x}, \sigma\right)}{\partial \theta^{l}} \frac{\partial \log p\left(x, y ; \mu_{x}, \sigma\right)}{\partial \theta^{l}},
$$

with $\theta \equiv\left(\theta^{1}, \theta^{2}\right) \stackrel{\text { def }}{=}\left(\mu_{x}, \sigma\right)$ and where $g_{l m}^{(2 D)}(\theta)$ has the following matrix representation,

$$
g_{l m}^{(2 D)}(\theta)=\frac{1}{\sigma^{2}}\left(\begin{array}{ll}
1 & 0 \\
0 & 4
\end{array}\right)
$$

Using (17), it follows that the non-vanishing Christoffel connection coefficients $\Gamma_{i j}^{k}$ are given by,

$$
\Gamma_{12}^{1}=\Gamma_{21}^{1}=-\frac{1}{\sigma}, \Gamma_{11}^{2}=\frac{1}{4 \sigma}, \Gamma_{22}^{2}=-\frac{1}{\sigma}
$$

The scalar curvature $\mathcal{R}^{(2 D)}$ of the probability distributions in (13) is given by,

$$
\mathcal{R}^{(2 D)}=g^{11}(\theta) R_{11}+g^{22}(\theta) R_{22}=-\frac{1}{2},
$$

with $g^{l m} g_{m k}=\delta_{k}^{l}$ and where the only non-vanishing Ricci curvature tensor component $R_{i j}$ is,

$$
R_{11}=-\frac{1}{4 \sigma^{2}}, R_{22}=-\frac{1}{\sigma^{2}}
$$

Observe that the only non-vanishing component of the Riemann curvature tensor $R_{\mu \nu \rho}^{\alpha}$ is,

$$
R_{212}^{1}=-\frac{1}{\sigma^{2}}
$$

thus,

$$
\mathcal{R}^{(2 D)} \equiv\left(R_{1212}+R_{2121}\right) g^{11}(\theta) g^{22}(\theta)=-\frac{1}{2},
$$

in agreement with Eq. (19). From (12) and (22), it turns out that the $3 D$ statistical model is globally more negatively curved than the $2 D$ statistical model.

\section{GEODESIC MOTION ON CURVED STATISTICAL MANIFOLDS}

In this Section, we present the geodesic paths on the curved statistical manifolds underlying the entropic dynamics of the three and two-dimensional Gaussian statistical models. Such paths are obtained by integrating the geodesic equations given by [39],

$$
\frac{d^{2} \theta^{k}(s)}{d \tau^{2}}+\Gamma_{l m}^{k}(\theta) \frac{d \theta^{l}}{d \tau} \frac{d \theta^{m}}{d \tau}=0
$$

where $\Gamma_{l m}^{k}(\theta)$ are the Christoffel symbols defined in (5).

\section{A. The Three-dimensional Case}

Substituting (6) into (23), the set of nonlinear and coupled ordinary differential equations in (23) reads,

$$
\begin{aligned}
& 0=\frac{d^{2} \mu_{x}}{d \tau^{2}}-\frac{2}{\sigma_{x}} \frac{d \mu_{x}}{d \tau} \frac{d \sigma_{x}}{d \tau}, \\
& 0=\frac{d^{2} \sigma_{x}}{d \tau^{2}}+\frac{1}{2 \sigma_{x}}\left(\frac{d \mu_{x}}{d \tau}\right)^{2}-\frac{1}{\sigma_{x}}\left(\frac{d \sigma}{d \tau}\right)^{2}, \\
& 0=\frac{d^{2} \sigma_{y}}{d \tau^{2}}-\frac{1}{\sigma_{y}}\left(\frac{d \sigma_{y}}{d \tau}\right)^{2} .
\end{aligned}
$$


A suitable family of geodesic paths fulfilling the geodesic equations above is given by (technical details appear in Appendix A),

$$
\mu_{x}(\tau)=\frac{\left(\mu_{0}+2 \sigma_{0}\right)\left[1+\exp \left(2 \sigma_{0} \lambda_{+}^{\prime} \tau\right)\right]-4 \sigma_{0}}{1+\exp \left(2 \sigma_{0} \lambda_{+}^{\prime} \tau\right)}, \sigma_{x}(\tau)=\frac{2 \sigma_{0} \exp \left(\sigma_{0} \lambda_{+}^{\prime} \tau\right)}{1+\exp \left(2 \sigma_{0} \lambda_{+}^{\prime} \tau\right)}
$$

and,

$$
\sigma_{y}(\tau)=\sigma_{0}^{\prime} \exp \left(-\lambda_{f} \tau\right)
$$

where $\mu_{0} \stackrel{\text { def }}{=} \mu_{x}(0), \sigma_{0} \stackrel{\text { def }}{=} \sigma_{x}(0), \sigma_{0}^{\prime} \stackrel{\text { def }}{=} \sigma_{y}(0), \varepsilon \stackrel{\text { def }}{=} \sigma_{y}\left(\tau_{f}\right), \lambda_{f} \stackrel{\text { def }}{=} \frac{1}{\tau_{f}} \log \left(\frac{\sigma_{0}}{\varepsilon}\right)$ and $\lambda_{+}^{\prime} \in \mathbb{R}^{+}$(see Appendix A).

\section{B. The Two-Dimensional Case}

Substituting (18) into (23), the set of nonlinear and coupled ordinary differential equations in (23) reads,

$$
\begin{aligned}
& 0=\frac{d^{2} \mu_{x}}{d \tau^{2}}-\frac{2}{\sigma} \frac{d \mu_{x}}{d \tau} \frac{d \sigma}{d \tau} \\
& 0=\frac{d^{2} \sigma}{d \tau^{2}}+\frac{1}{4 \sigma}\left(\frac{d \mu_{x}}{d \tau}\right)^{2}-\frac{1}{\sigma}\left(\frac{d \sigma}{d \tau}\right)^{2} .
\end{aligned}
$$

A suitable family of geodesic paths fulfilling the geodesic equations above is given by,

$$
\mu_{x}(\tau)=\frac{\left(\mu_{0}+2 \sigma_{0}\right)\left[1+\exp \left(2 \sigma_{0} \lambda_{+} \tau\right)\right]-4 \sigma_{0}}{1+\exp \left(2 \sigma_{0} \lambda_{+} \tau\right)}
$$

and,

$$
\sigma(\tau)=\frac{2 \sigma_{0} \exp \left(\sigma_{0} \lambda_{+} \tau\right)}{1+\exp \left(2 \sigma_{0} \lambda_{+} \tau\right)}
$$

where $\mu_{0} \stackrel{\text { def }}{=} \mu_{x}(0), \sigma_{0} \stackrel{\text { def }}{=} \sigma(0)$ and $\lambda_{+}=\frac{\lambda_{+}^{\prime}}{\sqrt{2}}$ belongs to $\mathbb{R}^{+}$(see Appendix A).

\section{INDICATORS OF CHAOTICITY}

In this Section, the chaotic properties of the information-constrained (entropic) dynamics on the underlying curved statistical manifolds are quantified by means of the Information Geometric Entropy and the Jacobi vector field intensity. The relevance of such quantities as suitable indicators of chaoticity was also investigated in [40].

\section{A. Information Geometric Entropy}

We point out that a suitable indicator of temporal complexity within the IGAC framework is provided by the IGE $\mathcal{S}_{\mathcal{M}_{s}}(\tau)$ [41],

$$
\mathcal{S}_{\mathcal{M}_{s}}(\tau) \stackrel{\text { def }}{=} \log \widetilde{\operatorname{vol}}\left[\mathcal{D}_{\theta}^{\text {(geodesic) }}(\tau)\right]
$$

The average dynamical statistical volume $\widetilde{\operatorname{vol}}\left[\mathcal{D}_{\Theta}^{\text {(geodesic) }}(\tau)\right]$ is defined as,

$$
\widetilde{\operatorname{vol}}\left[\mathcal{D}_{\theta}^{\text {(geodesic) }}(\tau)\right] \stackrel{\text { def }}{=} \lim _{\tau \rightarrow \infty}\left(\frac{1}{\tau} \int_{0}^{\tau} d \tau^{\prime} \operatorname{vol}\left[\mathcal{D}_{\theta}^{\text {(geodesic) }}\left(\tau^{\prime}\right)\right]\right) \text {, }
$$


where the "tilde" symbol denotes the operation of temporal average. For the sake of clarity, we point out that in the RHS of (31), we intend to preserve the temporal-dependence by considering the asymptotic leading term in the limit of $\tau$ approaching infinity. For a $n$-dimensional manifold, the volume $\operatorname{vol}\left[\mathcal{D}_{\theta}^{\text {(geodesic) }}\left(\tau^{\prime}\right)\right]$ in (31) is given by,

$$
\operatorname{vol}\left[\mathcal{D}_{\theta}^{\text {(geodesic) }}\left(\tau^{\prime}\right)\right] \stackrel{\text { def }}{=} \int_{\mathcal{D}_{\theta}^{\text {(geodesic) }}\left(\tau^{\prime}\right)} \rho_{\left(\mathcal{M}_{s}, g\right)}\left(\theta^{1}, \ldots, \theta^{n}\right) d^{n} \theta
$$

where $\rho_{\left(\mathcal{M}_{s}, g\right)}\left(\theta^{1}, \ldots, \theta^{n}\right)$ is the so-called Fisher density and equals the square root of the determinant of the metric tensor $g_{\mu \nu}(\theta)$,

$$
\rho_{\left(\mathcal{M}_{s}, g\right)}\left(\theta^{1}, \ldots, \theta^{n}\right) \stackrel{\text { def }}{=} \sqrt{g\left(\left(\theta^{1}, \ldots, \theta^{n}\right)\right)} .
$$

The integration space $\mathcal{D}_{\theta}^{\text {(geodesic) }}\left(\tau^{\prime}\right)$ in (32) is defined as follows,

$$
\mathcal{D}_{\theta}^{\text {(geodesic) }}\left(\tau^{\prime}\right) \stackrel{\text { def }}{=}\left\{\theta \equiv\left(\theta^{1}, \ldots, \theta^{n}\right): \theta^{k}(0) \leq \theta^{k} \leq \theta^{k}\left(\tau^{\prime}\right)\right\},
$$

where $k=1, . ., n$ and $\theta^{k} \equiv \theta^{k}(s)$ with $0 \leq s \leq \tau^{\prime}$ such that,

$$
\frac{d^{2} \theta^{k}(s)}{d s^{2}}+\Gamma_{l m}^{k} \frac{d \theta^{l}}{d s} \frac{d \theta^{m}}{d s}=0
$$

The integration space $\mathcal{D}_{\theta}^{\text {(geodesic) }}\left(\tau^{\prime}\right)$ in (34) is a $n$-dimensional subspace of the whole (permitted) parameter space $\mathcal{D}_{\theta}^{\text {(tot) }}$. The elements of $\mathcal{D}_{\theta}^{\text {(geodesic) }}\left(\tau^{\prime}\right)$ are the $n$-dimensional macrovariables $\{\theta\}$ whose components $\theta^{k}$ are bounded by specified limits of integration $\theta^{k}(0)$ and $\theta^{k}\left(\tau^{\prime}\right)$ with $k=1, . ., n$. The limits of integration are obtained via integration of the $n$-dimensional set of coupled nonlinear second order ordinary differential equations characterizing the geodesic equations. Formally, the IGE $\mathcal{S}_{\mathcal{M}_{s}}(\tau)$ is defined in terms of a averaged parametric $(n+1)$-fold integral $(\tau$ is the parameter) over the multidimensional geodesic paths connecting $\theta(0)$ to $\theta(\tau)$.

In the cases being investigated, using (25) and (26) it turns out that the $\mathcal{S}_{\mathcal{M}_{s}}^{(3 D \text { Model })}$ of the $3 D$ statistical model reads,

$$
\mathcal{S}_{\mathcal{M}_{s}}^{(3 D \text { Model })}=\log \mathcal{V}_{\mathcal{M}_{s}}^{(3 D \text { Model })}(\tau)
$$

with,

$$
\mathcal{V}_{\mathcal{M}_{s}}^{(3 D \text { Model })}(\tau)=\frac{1}{\sigma_{0}^{3} \lambda_{+}^{\prime}{ }^{2}} \frac{\exp \left(\sigma_{0} \lambda_{+}^{\prime} \tau\right)}{\tau}\left[\begin{array}{c}
\left(2 \sigma_{0}+\mu_{0}\right) \sigma_{0} \lambda_{f} \lambda_{+}^{\prime} \tau+\left(2 \sigma_{0}-\mu_{0}\right) \sigma_{0} \lambda_{f} \lambda_{+}^{\prime} \tau e^{-2 \sigma_{0} \lambda^{\prime} \tau}+ \\
-\left(\lambda_{f}+\lambda_{+}^{\prime} \sigma_{0} \ln \sigma_{0}^{\prime}\right)\left(2 \sigma_{0}+\mu_{0}\right)+ \\
-\left(\sigma_{0} \lambda_{+}^{\prime} \ln \sigma_{0}^{\prime}-\lambda_{f}\right)\left(2 \sigma_{0}-\mu_{0}\right) e^{-2 \sigma_{0} \tau \lambda^{\prime}}
\end{array}\right]
$$

In the asymptotic limit, we get

$$
\mathcal{V}_{\mathcal{M}_{s}}^{(3 D \text { Model })}(\tau) \stackrel{\tau \gg}{\approx}\left[\left(\frac{\lambda_{f}}{\lambda_{+}^{\prime}} \cdot \frac{\mu_{0}+2 \sigma_{0}}{\sigma_{0}^{2}}\right) \exp \left(\sigma_{0} \lambda_{+}^{\prime} \tau\right)\right],
$$

that is,

$$
\mathcal{S}_{\mathcal{M}_{s}}^{(3 D \text { Model })}(\tau) \stackrel{\tau \gg 1}{\approx} \lambda_{+}^{\prime} \tau
$$

Similarly, using (28) and (29), it follows that the $\mathcal{S}_{\mathcal{M}_{s}}^{(2 D \text { Model) }}$ of the $2 D$ model becomes,

$$
\mathcal{S}_{\mathcal{M}_{s}}^{(2 D \text { Model })}=\log \mathcal{V}_{\mathcal{M}_{s}}^{(2 D \text { Model })}(\tau)
$$

with,

$$
\mathcal{V}_{\mathcal{M}_{s}}^{(2 D \text { Model })}(\tau)=\frac{1}{\lambda_{+} \sigma_{0}^{2}} \frac{\left(\mu_{0}+2 \sigma_{0}\right)+\left(2 \sigma_{0}-\mu_{0}\right) \exp \left(-2 \sigma_{0} \lambda_{+} \tau\right)}{\tau \exp \left(-\sigma_{0} \lambda_{+} \tau\right)}
$$


In the asymptotic limit, we obtain

$$
\mathcal{V}_{\mathcal{M}_{s}}^{(2 D \text { Model })}(\tau) \stackrel{\tau \gg 1}{\approx}\left[\left(\frac{\mu_{0}+2 \sigma_{0}}{\sigma_{0}^{2} \lambda_{+}}\right) \frac{\exp \left(\sigma_{0} \lambda_{+} \tau\right)}{\tau}\right]
$$

and $\mathcal{S}_{\mathcal{M}_{s}}^{(2 D \text { Model })}$ becomes,

$$
\mathcal{S}_{\mathcal{M}_{s}}^{(2 D \text { Model })}(\tau) \stackrel{\tau \gg 1}{\approx} \lambda_{+} \tau
$$

Combining (43) and (39), it finally turns out that

$$
\mathcal{S}_{\mathcal{M}_{s}}^{(2 D \text { Model })}(\tau) \stackrel{\tau \gg 1}{\approx}\left[\left(\frac{\lambda_{+}}{\lambda_{+}^{\prime}}\right) \cdot \mathcal{S}_{\mathcal{M}_{s}}^{(3 D \text { Model })}(\tau)\right] \text { with } \frac{\lambda_{+}}{\lambda_{+}^{\prime}}=\frac{1}{\sqrt{2}}<1
$$

Eq. (44) is quite interesting since it quantitatively shows that the IGE is softened when approaching the twodimensional case from the three-dimensional case via the introduction of the macroscopic constraint (14) that is reminiscent of Heisenberg's minimum uncertainty relation where $x$ denotes the position of a particle and $y$ its conjugate momentum.

\section{B. Jacobi Vector Field Intensity}

The Jacobi-Levi-Civita (JLC) equation of geodesic deviation is a complicated second-order system of linear ordinary differential equations. It describes the geodesic spread on curved manifolds of a pair of nearby freely falling particles travelling on trajectories $\theta^{\rho}(\tau)$ and $\theta^{\prime \rho}(\tau) \stackrel{\text { def }}{=} \theta^{\rho}(\tau)+\delta \theta^{\rho}(\tau)$. The JLC equation is given by [42],

$$
\frac{D^{2} J^{k}}{D \tau^{2}}+R_{n m l}^{k} \frac{\partial \theta^{n}}{\partial \tau} J^{m} \frac{\partial \theta^{l}}{\partial \tau}=0
$$

with $k=1,2,3$ (in the three-dimensional case) and where the covariant derivative $\frac{D \theta^{\mu}(\tau)}{D \tau}$ along the curve $\theta^{\mu}(\tau)$ is defined as,

$$
\frac{D \theta^{\mu}(\tau)}{D \tau} \stackrel{\text { def }}{=} \frac{d \Theta^{\mu}(\tau)}{d \tau}+\Gamma_{\nu \rho}^{\mu} \frac{d \Theta^{\rho}}{d \tau} \Theta^{\nu}
$$

The Jacobi vector field components $J^{k}$ are given by,

$$
J^{k} \equiv \delta_{\lambda_{k}} \theta^{k} \stackrel{\text { def }}{=}\left(\frac{\partial \theta^{k}\left(\tau ; \lambda_{k}\right)}{\partial \lambda_{k}}\right)_{\tau} \delta \lambda_{k}
$$

and $R_{\alpha \beta \gamma \delta}$ is the Riemann curvature tensor defined as [42],

$$
R_{\mu \nu \rho}^{\alpha} \stackrel{\text { def }}{=} \partial_{\nu} \Gamma_{\mu \rho}^{\alpha}-\partial_{\rho} \Gamma_{\mu \nu}^{\alpha}+\Gamma_{\beta \nu}^{\alpha} \Gamma_{\mu \rho}^{\beta}-\Gamma_{\beta \rho}^{\alpha} \Gamma_{\mu \nu}^{\beta}
$$

The covariant derivative $\frac{D^{2} J^{\mu}}{D \tau^{2}}$ in (45) is defined as [43],

$$
\frac{D^{2} J^{\mu}}{D \tau^{2}}=\frac{d^{2} J^{\mu}}{d \tau^{2}}+2 \Gamma_{\alpha \beta}^{\mu} \frac{d J^{\alpha}}{d \tau} \frac{d \Theta^{\beta}}{d \tau}+\Gamma_{\alpha \beta}^{\mu} J^{\alpha} \frac{d^{2} \Theta^{\beta}}{d \tau^{2}}+\Gamma_{\alpha \beta, \nu}^{\mu} \frac{d \Theta^{\nu}}{d \tau} \frac{d \Theta^{\beta}}{d \tau} J^{\alpha}+\Gamma_{\alpha \beta}^{\mu} \Gamma_{\rho \sigma}^{\alpha} \frac{d \Theta^{\sigma}}{d \tau} \frac{d \Theta^{\beta}}{d \tau} J^{\rho} .
$$

Equation (45) forms a system of coupled ordinary differential equations linear in the components of the deviation vector field (47) but nonlinear in derivatives of the metric tensor $g_{i j}(\theta)$. It describes the linearized geodesic flow: the linearization ignores the relative velocity of the geodesics. When the geodesics are neighboring but their relative velocity is arbitrary, the corresponding geodesic deviation equation is the so-called generalized Jacobi equation [44]. The nonlinearity is due to the existence of velocity-dependent terms in the system. Neighboring geodesics accelerate relative to each other with a rate directly measured by the curvature tensor $R_{\alpha \beta \gamma \delta}$.

Omitting technical details (see Appendix B) and setting $\Lambda_{3 D} \stackrel{\text { def }}{=} \lambda_{3 D} \sigma_{0}$ with $\lambda_{3 D}=\lambda_{+}^{\prime}$, the JLC equation for $J^{1}$ becomes,

$$
\frac{d^{2} J^{1}}{d \tau^{2}}+2 \Lambda_{3 D} \frac{d J^{1}}{d \tau}-8 \Lambda_{3 D} \exp \left(-\Lambda_{3 D} \tau\right) \frac{d J^{2}}{d \tau}-8 \Lambda_{3 D} \exp \left(-2 \Lambda_{3 D} \tau\right) J^{1}-8 \Lambda_{3 D}^{2} \exp \left(-\Lambda_{3 D} \tau\right) J^{2}=0
$$


Similarly, it can be shown that the JLC equations for $J^{2}$ and $J^{3}$ read,

$$
\frac{d^{2} J^{2}}{d \tau^{2}}+4 \Lambda_{3 D} \exp \left(-\Lambda_{3 D} \tau\right) \frac{d J^{1}}{d \tau}+2 \Lambda_{3 D} \frac{d J^{2}}{d \tau}+\Lambda_{3 D}^{2} J^{2}=0
$$

and,

$$
\frac{d^{2} J^{3}}{d \tau^{2}}+2 \lambda_{f} \frac{d J^{3}}{d \tau}+\lambda_{f}^{2} J^{3}=0
$$

respectively. In the asymptotic limit, it can be shown that the three equations to integrate become (see Appendix B)

$$
\begin{aligned}
\frac{d^{2} J^{1}}{d \tau^{2}}+2 \Lambda_{3 D} \frac{d J^{1}}{d \tau} & =0, \\
\frac{d^{2} J^{2}}{d \tau^{2}}+2 \Lambda_{3 D} \frac{d J^{2}}{d \tau}+\Lambda_{3 D}^{2} J^{2} & =0, \\
\frac{d^{2} J^{3}}{d \tau^{2}}+2 \lambda_{f} \frac{d J^{3}}{d \tau}+\lambda_{f}^{2} J^{3} & =0 .
\end{aligned}
$$

The asymptotic solutions are given by,

$$
\begin{aligned}
& J^{1}(\tau)=C_{1}^{(1)}+C_{2}^{(1)} \exp \left(-2 \Lambda_{3 D} \tau\right) \\
& J^{2}(\tau)=C_{1}^{(2)} \exp \left(-\Lambda_{3 D} \tau\right)+C_{2}^{(2)} \tau \exp \left(-\Lambda_{3 D} \tau\right), \\
& J^{3}(\tau)=C_{1}^{(3)} \exp \left(-\lambda_{f} \tau\right)+C_{2}^{(3)} \tau \exp \left(-\lambda_{f} \tau\right),
\end{aligned}
$$

where $C_{k^{\prime}}^{(k)}$ with $k=1,2,3$ and $k^{\prime}=1,2$ are real integration constants. The Jacobi vector field intensity $J_{\mathcal{M}_{s}}^{(3 D)}(\tau)$ is defined as,

$$
J_{\mathcal{M}_{s}}^{(3 D)}(\tau) \stackrel{\text { def }}{=}\left[\frac{\left[J^{1}(\tau)\right]^{2}}{\sigma_{x}^{2}(\tau)}+\frac{2\left[J^{2}(\tau)\right]^{2}}{\sigma_{x}^{2}(\tau)}+\frac{2\left[J^{3}(\tau)\right]^{2}}{\sigma_{y}^{2}(\tau)}\right]^{\frac{1}{2}}
$$

Eqs. (25), (26) and (54) imply that,

$$
\frac{\left[J^{1}(\tau)\right]^{2}}{\sigma_{x}^{2}(\tau)} \approx \frac{\left[C_{1}^{(1)}\right]^{2}}{4 \sigma_{0}^{2}} \exp \left(2 \Lambda_{3 D} \tau\right), \frac{2\left[J^{2}(\tau)\right]^{2}}{\sigma_{x}^{2}(\tau)} \approx \frac{\left[C_{2}^{(2)}\right]^{2}}{2 \sigma_{0}^{2}} \tau^{2} \text { and, } \frac{2\left[J^{3}(\tau)\right]^{2}}{\sigma_{y}^{2}(\tau)} \approx \frac{2\left[C_{2}^{(3)}\right]^{2}}{\sigma_{0}^{\prime 2}} \tau^{2} .
$$

It finally follows that $J_{\mathcal{M}_{s}}^{(3 D)}(\tau)$ reads,

$$
J_{\mathcal{M}_{s}}^{(3 D)}(\tau) \approx \frac{C_{1}^{(1)}}{2 \sigma_{0}} \exp \left(\Lambda_{3 D} \tau\right)
$$

Consider now the two-dimensional statistical model. Omitting technical details (see Appendix B) and setting $\Lambda_{2 D} \stackrel{\text { def }}{=}$ $\lambda_{2 D} \sigma_{0}$ with $\lambda_{2 D}=\lambda_{+}$, the two JLC equations for $J^{1}$ and $J^{2}$ read,

$$
\frac{d^{2} J^{1}}{d \tau^{2}}+2 \Lambda_{2 D} \frac{d J^{1}}{d \tau}-8 \Lambda_{2 D} \exp \left(-\Lambda_{2 D} \tau\right) \frac{d J^{2}}{d \tau}-4 \Lambda_{2 D}^{2} \exp \left(-2 \Lambda_{2 D} \tau\right) J^{1}-8 \Lambda_{2 D}^{2} \exp \left(-\Lambda_{2 D} \tau\right) J^{2}=0
$$

and,

$$
\frac{d^{2} J^{2}}{d \tau^{2}}+2 \Lambda_{2 D} \exp \left(-\Lambda_{2 D} \tau\right) \frac{d J^{1}}{d \tau}+2 \Lambda_{2 D} \frac{d J^{2}}{d \tau}+\Lambda_{2 D}^{2} J^{2}=0
$$


respectively. Following the line of reasoning provided for the three-dimensional case, the asymptotic integration of (58) and (59) lead to

$$
J^{1}(\tau)=C_{1}^{(1)}+C_{2}^{(1)} \exp \left(-2 \Lambda_{2 D} \tau\right)
$$

and,

$$
J^{2}(\tau)=C_{1}^{(2)} \exp \left(-\Lambda_{2 D} \tau\right)+C_{2}^{(2)} \tau \exp \left(-\Lambda_{2 D} \tau\right)
$$

respectively where $C_{k^{\prime}}^{(k)}$ with $k=1,2$ and $k^{\prime}=1,2$ are real integration constants. The Jacobi vector field intensity $J_{\mathcal{M}_{s}}^{(2 D)}(\tau)$ is defined as,

$$
J_{\mathcal{M}_{s}}^{(2 D)}(\tau) \stackrel{\text { def }}{=}\left[\frac{\left[J^{1}(\tau)\right]^{2}}{\sigma^{2}(\tau)}+\frac{4\left[J^{2}(\tau)\right]^{2}}{\sigma^{2}(\tau)}\right]^{\frac{1}{2}} .
$$

Eqs. (29), (60) and (61) imply that,

$$
\frac{\left[J^{1}(\tau)\right]^{2}}{\sigma^{2}(\tau)} \approx \frac{\left[C_{1}^{(1)}\right]^{2}}{4 \sigma_{0}^{2}} \exp \left(2 \Lambda_{2 D} \tau\right), \frac{4\left[J^{2}(\tau)\right]^{2}}{\sigma^{2}(\tau)} \approx \frac{\left[C_{2}^{(2)}\right]^{2}}{\sigma_{0}^{2}} \tau^{2} .
$$

It then follows that,

$$
J_{\mathcal{M}_{s}}^{(2 D)}(\tau) \approx \frac{C_{1}^{(1)}}{2 \sigma_{0}} \exp \left(\Lambda_{2 D} \tau\right)
$$

Thus, we have shown that in the asymptotic limit,

$$
J_{\mathcal{M}_{s}}^{(3 D)}(\tau) \approx \frac{C_{1}^{(1)}}{2 \sigma_{0}} \exp \left(\Lambda_{3 D} \tau\right) \text { and, } J_{\mathcal{M}_{s}}^{(2 D)}(\tau) \approx \frac{C_{1}^{(1)}}{2 \sigma_{0}} \exp \left(\Lambda_{2 D} \tau\right),
$$

that is,

$$
J_{\mathcal{M}_{s}}^{(2 D)}(\tau) \approx e^{-\left(\Lambda_{3 D}-\Lambda_{2 D}\right) \tau} \cdot J_{\mathcal{M}_{s}}^{(3 D)}(\tau) \text { with } \Lambda_{3 D}-\Lambda_{2 D}>0 .
$$

Eq. (66) is quite enlightening since it shows that the Jacobi vector field intensity is softened when approaching the two-dimensional case from the three-dimensional case via the introduction of the quantum-like macroscopic constraint (14).

We emphasize that our main findings (see Eqs. (44) and (66) ) presented in this work are limited to the asymptotic behavior at infinity on the $\tau$-scale of the selected indicators of chaoticity, that is, the IGE and the JLC vector field intensity. However, recall that in quantum chaos, the shortest random time scale (or Ehrenfest time scale) $t_{r}$ is approximately given by [45],

$$
t_{r} \approx \frac{1}{\lambda} \log \left(\frac{S}{\hbar}\right)
$$

where $\lambda$ is the maximum Lyapunov exponent of the system, $\hbar \stackrel{\text { def }}{=} \frac{h}{2 \pi}$ is the Dirac constant, $h$ is the Planck constant and $S \simeq \int p d q$ is a characteristic reference value of a classical action. This time scale is especially important because on time scales of this order, the complete transition from quantum to classical dynamical chaos is allowed. Stated otherwise, on time scales of this order, quantum motion is similar to the classical one including the exponential instability [46]. Therefore, it would be worthwhile investigating whether or not for the statistical dynamical Gaussian models studied here, there is any information geometric analogue of the standard random time scale, say $\tau_{r}$, such that one may find softening effects on a $\tau$-scale longer than a finite $\tau_{r}$. This investigation would present two delicate points: first, we would need the JLC-equation analysis on finite- $\tau$ scales which would be extremely difficult from a computational point of view; second, our geodesic affine parameter $\tau$ is not the conventional time $t$. Therefore, unless the connection between $\tau$ and $t$ is clearly specified, this analogy would be admittedly vague. However, we believe that this investigation may be successfully tackled within the IGAC framework at least in specific cases, for instance for conservative chaotic Hamiltonian systems. In such cases, there is a neat connection between the standard time-scale and the geodesic parameter-scale and the difficulties in the integration of the JLC equation may not be insurmountable [47]. We will examine this issue in forthcoming works. 


\section{CONCLUDING REMARKS}

In this article, we studied both the information geometry and the entropic dynamics of a $3 D$ Gaussian statistical model. We then compared our analysis to that of a $2 D$ Gaussian statistical model obtained from the higher-dimensional model via introduction of an additional information constraint that resembles the quantum mechanical canonical minimum uncertainty relation. We showed that the chaoticity (temporal complexity) of the $2 D$ Gaussian statistical model, quantified by means of the Information Geometric Entropy (IGE) and the Jacobi vector field intensity, is softened with respect to the chaoticity of the $3 D$ Gaussian statistical model. Specifically, Eq. (44) shows that the IGE is softened when approaching the two-dimensional case from the three-dimensional case via the introduction of the macroscopic constraint (14) that resembles the quantum mechanical canonical minimum uncertainty relation. Furthermore, Eq. (66) confirms that also the Jacobi vector field intensity is softened when approaching the twodimensional case from the three-dimensional case via the introduction of the macroscopic constraint (14).

We stress that our information geometric analysis could be further generalized to accommodate non minimum uncertainty-like relations. However, such extension requires a more delicate analysis where Maximum relative Entropy methods are used to process information in the presence of inequality constraints [48]. Our work is especially relevant for the quantification of soft chaos effects in entropic dynamical models used to describe actual physical systems when only incomplete knowledge about them is available [49]. Furthermore, although we are aware that our analysis is not manifestly "quantum", our findings lead us to support the conjecture that quantum chaos is ultimately weaker than classical chaos because of Heisenberg's uncertainty relation, the most important difference between classical and quantum physics. Of course, a deeper analysis is needed and we leave it for future investigations.

\section{Acknowledgments}

The research of C. Cafaro, C. Lupo and S. Mancini has received funding from the European Commission's Seventh Framework Programme (FP7/2007-2013) under grant agreements no. 213681.

\section{Appendix A: Integration of Geodesic Equations}

We show here the details leading to the geodesic paths presented in Section III.

Consider the following set of coupled nonlinear differential equations,

$$
\frac{d^{2} \mu_{x}}{d \tau^{2}}-\frac{2}{\sigma_{x}} \frac{d \mu_{x}}{d \tau} \frac{d \sigma_{x}}{d \tau}=0 \text { and, } \frac{d^{2} \sigma_{x}}{d \tau^{2}}+\frac{1}{2 \sigma_{x}}\left(\frac{d \mu_{x}}{d \tau}\right)^{2}-\frac{1}{\sigma_{x}}\left(\frac{d \sigma_{x}}{d \tau}\right)^{2}=0 .
$$

Setting $\dot{\mu}_{x} \stackrel{\text { def }}{=} \frac{d \mu_{x}}{d \tau}$ and $\dot{\sigma}_{x} \stackrel{\text { def }}{=} \frac{d \sigma_{x}(\tau)}{d \tau}$, the two equations in (A1) become,

$$
\ddot{\mu}_{x}-2 \frac{\dot{\sigma}_{x}}{\sigma_{x}} \dot{\mu}_{x}=0 \text { and, } \ddot{\sigma}_{x}+\frac{1}{2 \sigma_{x}} \dot{\mu}_{x}^{2}-\frac{\dot{\sigma}_{x}^{2}}{\sigma_{x}}=0 .
$$

From (A2) we observe that

$$
\frac{\ddot{\mu}_{x}}{\dot{\mu}_{x}}=2 \frac{\dot{\sigma}_{x}}{\sigma_{x}} .
$$

Therefore, we have that

$$
\dot{\mu}_{x}(\tau)=A_{1} \sigma_{x}^{2}(\tau),
$$

with $A_{1} \in \mathbb{R}$. Substituting (4ㄴ in the second equation in (A2), we get

$$
\sigma_{x} \ddot{\sigma}_{x}-\dot{\sigma}_{x}^{2}+\frac{A_{1}^{2}}{2} \sigma_{x}^{4}=0 .
$$

Our goal is to integrate Eq. (A5), find $\sigma_{x}(\tau)$ and finally compute $\mu_{x}(\tau)$ using (A4). For the sake of simplicity, let us put $\sigma_{x}(\tau) \equiv y(\tau)$ and $a \stackrel{\text { def }}{=} \frac{A_{1}^{2}}{2} \in \mathbb{R}_{0}^{+}$. Then, Eq. (A5) becomes

$$
y \ddot{y}-\dot{y}^{2}+a y^{4}=0 .
$$


As a first change of variables, let us set

$$
y(\tau) \stackrel{\text { def }}{=} \frac{d x(\tau)}{d \tau}=\dot{x}(\tau)
$$

Substituting (A7) into (A6), we obtain

$$
\ddot{x} \dddot{x}-\ddot{x}^{2}+a \dot{x}^{4}=0 .
$$

As a second change of variables, let us set

$$
\dot{x}=\frac{d x(\tau)}{d \tau} \stackrel{\text { def }}{=} z(x) .
$$

Therefore, it follows that

$$
\ddot{x}=z z^{\prime}, \dddot{x}=\left(z^{\prime \prime} z+z^{\prime 2}\right) z, z^{\prime}=\frac{d z(x)}{d x} .
$$

Substituting (A10) and (A9) in (A8), we get

$$
z^{\prime \prime}+a z=0
$$

Integration of (A11) yields,

$$
z(x)=C_{1} \sin \left(\sqrt{a} x+C_{2}\right)
$$

where the integration constant coefficients $C_{1}$ and $C_{2}$ belong to $\mathbb{R}$. Recalling that $\dot{x}=z(x)$, we get

$$
\int^{x} \frac{1}{C_{1} \sin \left(\sqrt{a} x^{\prime}+C_{2}\right)} d x^{\prime}=\int^{\tau} d \tau^{\prime} .
$$

Upon integration, (A13) becomes

$$
\frac{1}{\sqrt{a} C_{1}} \log \left[\tan \left(\frac{\sqrt{a} x+C_{2}}{2}\right)\right]=\tau+C_{3}
$$

with $C_{3} \in \mathbb{R}$. Solving for $x=x(\tau)$, we finally obtain

$$
x(\tau)=\frac{1}{\sqrt{a}}\left\{2 \arctan \left(\exp \left[\sqrt{a} C_{1}\left(\tau+C_{3}\right)\right]\right)-C_{2}\right\} .
$$

Finally, $\sigma_{x}(\tau)$ reads

$$
\sigma_{x}(\tau)=\frac{2 c_{1} \exp \left(c_{1} \sqrt{c_{2}} \tau+c_{1} c_{3} \sqrt{c_{2}}\right)}{1+\exp \left(2 c_{1} \sqrt{c_{2}} \tau+2 c_{1} c_{3} \sqrt{c_{2}}\right)}
$$

where we have set $c_{1}=C_{1}, c_{2}=a \stackrel{\text { def }}{=} \frac{A_{1}^{2}}{2}$ and $c_{3}=C_{3}$. It is straightforward to verify that indeed $\sigma_{x}(\tau)$ in (A16) satisfies the nonlinear differential equation (A6). Finally, we can compute $\mu_{x}(\tau)$ using (A4). It follows that,

$$
\mu_{x}(\tau)=\sqrt{4 a} \int^{\tau} \sigma_{x}^{2}\left(\tau^{\prime}\right) d \tau^{\prime}=\frac{c_{4}\left[1+\exp \left(2 c_{1} \sqrt{c_{2}} \tau+2 c_{1} c_{3} \sqrt{c_{2}}\right)\right]-4 c_{1}}{1+\exp \left(2 c_{1} \sqrt{c_{2}} \tau+2 c_{1} c_{3} \sqrt{c_{2}}\right)}
$$

Therefore, the geodesic paths are given by

$$
\mu_{x}(\tau)=\frac{c_{4}\left[1+\exp \left(2 c_{1} \sqrt{c_{2}} \tau+2 c_{1} c_{3} \sqrt{c_{2}}\right)\right]-4 c_{1}}{1+\exp \left(2 c_{1} \sqrt{c_{2}} \tau+2 c_{1} c_{3} \sqrt{c_{2}}\right)} \text { and, } \sigma_{x}(\tau)=\frac{2 c_{1} \exp \left(c_{1} \sqrt{c_{2}} \tau+c_{1} c_{3} \sqrt{c_{2}}\right)}{1+\exp \left(2 c_{1} \sqrt{c_{2}} \tau+2 c_{1} c_{3} \sqrt{c_{2}}\right)}
$$

As working hypothesis, we consider geodesic paths with $c_{3}=0$ and assume that the initial conditions given by $\mu_{x}(0)=\mu_{0}$ and $\sigma(0)=\sigma_{0}$. The geodesics in (A18) become,

$$
\mu_{x}(\tau)=\frac{\left(\mu_{0}+2 \sigma_{0}\right)\left[1+\exp \left(2 \sigma_{0} \lambda_{+}^{\prime} \tau\right)\right]-4 \sigma_{0}}{1+\exp \left(2 \sigma_{0} \lambda_{+}^{\prime} \tau\right)}, \sigma_{x}(\tau)=\frac{2 \sigma_{0} \exp \left(\sigma_{0} \lambda_{+}^{\prime} \tau\right)}{1+\exp \left(2 \sigma_{0} \lambda_{+}^{\prime} \tau\right)}
$$


where $\lambda_{+}^{\prime}=\sqrt{c_{2}}=\sqrt{a}>0$ since $a \stackrel{\text { def }}{=} \frac{A_{1}^{2}}{2}>0$.

Using the same tricks (invertible changes of variables) presented above, it becomes straightforward integrating the last differential equation characterizing the three dimensional case,

$$
\sigma_{y} \ddot{\sigma}_{y}-\dot{\sigma}_{y}^{2}=0
$$

Its solution $\sigma_{y}(\tau)$ is given by,

$$
\sigma_{y}(\tau)=\sigma_{0}^{\prime} \exp \left(-\lambda_{f} \tau\right)
$$

with $\sigma_{y}(0)=\sigma_{0}^{\prime}, \sigma_{y}\left(\tau_{f}\right)=\varepsilon$ and $\lambda_{f} \stackrel{\text { def }}{=} \frac{1}{\tau_{f}} \log \left(\frac{\sigma_{0}^{\prime}}{\varepsilon}\right)$.

Finally, following the line of reasoning presented above and assuming the very same working hypothesis and initial conditions, it turns out that the geodesic paths in the two-dimensional case read,

$$
\mu_{x}(\tau)=\frac{\left(\mu_{0}+2 \sigma_{0}\right)\left[1+\exp \left(2 \sigma_{0} \lambda_{+} \tau\right)\right]-4 \sigma_{0}}{1+\exp \left(2 \sigma_{0} \lambda_{+} \tau\right)}, \sigma(\tau)=\frac{2 \sigma_{0} \exp \left(\sigma_{0} \lambda_{+} \tau\right)}{1+\exp \left(2 \sigma_{0} \lambda_{+} \tau\right)},
$$

where $\lambda_{+}=\sqrt{a}>0$ since $a \stackrel{\text { def }}{=} \frac{A_{1}^{2}}{4}>0$.

\section{Appendix B: Integration of Jacobi-Levi-Civita Equations}

We present here few technical details relative to the JLC equation analysis introduced in Section IV.

The JLC equations in the three-dimensional case are three. The JLC equation for $J^{1}$ reads,

$$
\frac{D^{2} J^{1}}{D \tau^{2}}-\frac{1}{\sigma_{x}^{2}}\left[J^{1}\left(\frac{d \sigma_{x}}{d \tau}\right)^{2}-J^{2} \frac{d \mu_{x}}{d \tau} \frac{d \sigma_{x}}{d \tau}\right]=0
$$

where we have used the relation $R_{212}^{1}=-\frac{1}{\sigma_{x}^{2}}$. Recall that,

$$
\frac{D^{2} J^{\mu}}{D \tau^{2}}=\frac{d^{2} J^{\mu}}{d \tau^{2}}+2 \Gamma_{\alpha \beta}^{\mu} \frac{d J^{\alpha}}{d \tau} \frac{d \theta^{\beta}}{d \tau}+\Gamma_{\alpha \beta}^{\mu} J^{\alpha} \frac{d^{2} \theta^{\beta}}{d \tau^{2}}+\partial_{\nu} \Gamma_{\alpha \beta}^{\mu} \frac{d \theta^{\nu}}{d \tau} \frac{d \theta^{\beta}}{d \tau} J^{\alpha}+\Gamma_{\alpha \beta}^{\mu} \Gamma_{\rho \sigma}^{\alpha} \frac{d \theta^{\sigma}}{d \tau} \frac{d \theta^{\beta}}{d \tau} J^{\rho} .
$$

For $J^{1}$ we get,

$$
\begin{aligned}
\frac{D^{2} J^{1}}{D \tau^{2}} & =\frac{d^{2} J^{1}}{d \tau^{2}}+2 \Gamma_{12}^{1}\left(\frac{d J^{1}}{d \tau} \frac{d \theta^{2}}{d \tau}+\frac{d J^{2}}{d \tau} \frac{d \theta^{1}}{d \tau}\right)+\Gamma_{12}^{1}\left(J^{1} \frac{d^{2} \theta^{2}}{d \tau^{2}}+J^{2} \frac{d^{2} \theta^{1}}{d \tau^{2}}\right)+ \\
& +\partial_{2} \Gamma_{12}^{1}\left[\left(\frac{d \theta^{2}}{d \tau}\right)^{2} J^{1}+\frac{d \theta^{1}}{d \tau} \frac{d \theta^{2}}{d \tau} J^{2}\right]+\left(\Gamma_{12}^{1}\right)^{2}\left(\frac{d \theta^{2}}{d \tau}\right)^{2} J^{1}+2\left(\Gamma_{12}^{1}\right)^{2} \frac{d \theta^{1}}{d \tau} \frac{d \theta^{2}}{d \tau}+\Gamma_{12}^{1} \Gamma_{11}^{2}\left(\frac{d \theta^{1}}{d \tau}\right)^{2} J^{1}
\end{aligned}
$$

where we have used the identity,

$$
\Gamma_{\alpha \beta}^{1} \Gamma_{\rho \sigma}^{\alpha} \frac{d \theta^{\sigma}}{d \tau} \frac{d \theta^{\beta}}{d \tau} J^{\rho}=\left(\Gamma_{12}^{1}\right)^{2}\left(\frac{d \theta^{2}}{d \tau}\right)^{2} J^{1}+2\left(\Gamma_{12}^{1}\right)^{2} \frac{d \theta^{1}}{d \tau} \frac{d \theta^{2}}{d \tau}+\Gamma_{12}^{1} \Gamma_{11}^{2}\left(\frac{d \theta^{1}}{d \tau}\right)^{2} J^{1} .
$$

since $\Gamma_{12}^{1}=\Gamma_{21}^{1}=\Gamma_{22}^{2}$. From (B1) and (B3) and after some algebra, we get

$$
\begin{aligned}
0 & =\frac{d^{2} J^{1}}{d \tau^{2}}+2 \Gamma_{12}^{1} \frac{d \sigma_{x}}{d \tau} \frac{d J^{1}}{d \tau}+2 \Gamma_{12}^{1} \frac{d \mu_{x}}{d \tau} \frac{d J^{2}}{d \tau}+ \\
& +J^{1}\left[\Gamma_{12}^{1} \frac{d^{2} \sigma_{x}}{d \tau^{2}}+\partial_{2} \Gamma_{12}^{1}\left(\frac{d \sigma_{x}}{d \tau}\right)^{2}+\left(\Gamma_{12}^{1}\right)^{2}\left(\frac{d \sigma_{x}}{d \tau}\right)^{2}+\Gamma_{12}^{1} \Gamma_{11}^{2}\left(\frac{d \mu_{x}}{d \tau}\right)^{2}+R_{212}^{1}\left(\frac{d \sigma_{x}}{d \tau}\right)^{2}\right]+ \\
& +J^{2}\left[\Gamma_{12}^{1} \frac{d^{2} \mu_{x}}{d \tau^{2}}+\partial_{2} \Gamma_{12}^{1} \frac{d \mu_{x}}{d \tau} \frac{d \sigma_{x}}{d \tau}+2\left(\Gamma_{12}^{1}\right)^{2} \frac{d \mu_{x}}{d \tau} \frac{d \sigma_{x}}{d \tau}-R_{212}^{1} \frac{d \mu_{x}}{d \tau} \frac{d \sigma_{x}}{d \tau}\right]
\end{aligned}
$$


that is,

$$
\begin{aligned}
0 & =\frac{d^{2} J^{1}}{d \tau^{2}}+2 \Gamma_{12}^{1} \frac{d \sigma_{x}}{d \tau} \frac{d J^{1}}{d \tau}+2 \Gamma_{12}^{1} \frac{d \mu_{x}}{d \tau} \frac{d J^{2}}{d \tau}+ \\
& +J^{1}\left[\Gamma_{12}^{1} \frac{d^{2} \sigma_{x}}{d \tau^{2}}+\left(\partial_{2} \Gamma_{12}^{1}+\left(\Gamma_{12}^{1}\right)^{2}+R_{212}^{1}\right)\left(\frac{d \sigma_{x}}{d \tau}\right)^{2}+\Gamma_{12}^{1} \Gamma_{11}^{2}\left(\frac{d \mu_{x}}{d \tau}\right)^{2}\right]+ \\
& +J^{2}\left[\Gamma_{12}^{1} \frac{d^{2} \mu_{x}}{d \tau^{2}}+\left(\partial_{2} \Gamma_{12}^{1}+2\left(\Gamma_{12}^{1}\right)^{2}-R_{212}^{1}\right) \frac{d \mu_{x}}{d \tau} \frac{d \sigma_{x}}{d \tau}\right]
\end{aligned}
$$

Observing that,

$$
\left(\Gamma_{12}^{1}\right)^{2}=\frac{1}{\sigma_{x}^{2}}, R_{212}^{1}=-\frac{1}{\sigma_{x}^{2}} \text { and }, \partial_{2} \Gamma_{12}^{1}+2\left(\Gamma_{12}^{1}\right)^{2}-R_{212}^{1}=\frac{4}{\sigma_{x}^{2}}
$$

Eq. (B6) becomes,

$$
\begin{aligned}
0 & =\frac{d^{2} J^{1}}{d \tau^{2}}+2 \Gamma_{12}^{1} \frac{d \sigma_{x}}{d \tau} \frac{d J^{1}}{d \tau}+2 \Gamma_{12}^{1} \frac{d \mu_{x}}{d \tau} \frac{d J^{2}}{d \tau}+ \\
& +J^{1}\left[\Gamma_{12}^{1} \frac{d^{2} \sigma_{x}}{d \tau^{2}}+\partial_{2} \Gamma_{12}^{1}\left(\frac{d \sigma_{x}}{d \tau}\right)^{2}+\Gamma_{12}^{1} \Gamma_{11}^{2}\left(\frac{d \mu_{x}}{d \tau}\right)^{2}\right]+ \\
& +J^{2}\left[\Gamma_{12}^{1} \frac{d^{2} \mu_{x}}{d \tau^{2}}+\frac{4}{\sigma_{x}^{2}} \frac{d \mu_{x}}{d \tau} \frac{d \sigma_{x}}{d \tau}\right]
\end{aligned}
$$

that is,

$$
\begin{aligned}
0 & =\frac{d^{2} J^{1}}{d \tau^{2}}+\left(-\frac{2}{\sigma_{x}} \frac{d \sigma_{x}}{d \tau}\right) \frac{d J^{1}}{d \tau}+\left(-\frac{2}{\sigma_{x}} \frac{d \mu_{x}}{d \tau}\right) \frac{d J^{2}}{d \tau}+ \\
& +J^{1}\left[-\frac{1}{\sigma_{x}} \frac{d^{2} \sigma_{x}}{d \tau^{2}}+\frac{1}{\sigma_{x}^{2}}\left(\frac{d \sigma_{x}}{d \tau}\right)^{2}-\frac{1}{2 \sigma_{x}^{2}}\left(\frac{d \mu_{x}}{d \tau}\right)^{2}\right]+ \\
& +J^{2}\left[-\frac{1}{\sigma_{x}} \frac{d^{2} \mu_{x}}{d \tau^{2}}+\frac{4}{\sigma_{x}^{2}} \frac{d \mu_{x}}{d \tau} \frac{d \sigma_{x}}{d \tau}\right]
\end{aligned}
$$

At this point we recall that the geodesic paths $\mu_{x}(\tau)$ and $\sigma_{x}(\tau)$ for the $3 D$ statistical model are given by,

$$
\mu_{x}(\tau)=\frac{\left(\mu_{0}+2 \sigma_{0}\right)\left[1+\exp \left(2 \sqrt{a} \sigma_{0} \tau\right)\right]-4 \sigma_{0}}{1+\exp \left(2 \sqrt{a} \sigma_{0} \tau\right)}
$$

and,

$$
\sigma_{x}(\tau)=\frac{2 \sigma_{0} \exp \left(\sqrt{a} \sigma_{0} \tau\right)}{1+\exp \left(2 \sqrt{a} \sigma_{0} \tau\right)}
$$


respectively. Therefore, in the asymptotic limit we get

$$
\begin{aligned}
& \mu_{x}(\tau) \approx \text { const., } \sigma_{x}(\tau) \approx 2 \sigma_{0} \exp \left(-\sqrt{a} \sigma_{0} \tau\right), \\
& \frac{d \sigma_{x}(\tau)}{d \tau} \approx-2 \sqrt{a} \sigma_{0}^{2} \exp \left(-\sqrt{a} \sigma_{0} \tau\right),\left(\frac{d \sigma_{x}(\tau)}{d \tau}\right)^{2} \approx 4 a \sigma_{0}^{4} \exp \left(-2 \sqrt{a} \sigma_{0} \tau\right), \\
& \frac{d \mu_{x}(\tau)}{d \tau} \approx 8 \sqrt{a} \sigma_{0}^{2} \exp \left(-2 \sqrt{a} \sigma_{0} \tau\right),\left(\frac{d \mu_{x}(\tau)}{d \tau}\right)^{2} \approx 64 a \sigma_{0}^{4} \exp \left(-4 \sqrt{a} \sigma_{0} \tau\right), \\
& \frac{d^{2} \mu_{x}(\tau)}{d \tau^{2}} \approx-16 a \sigma_{0}^{3} \exp \left(-2 \sqrt{a} \sigma_{0} \tau\right), \frac{d^{2} \sigma_{x}(\tau)}{d \tau^{2}} \approx 2 a \sigma_{0}^{3} \exp \left(-\sqrt{a} \sigma_{0} \tau\right) .
\end{aligned}
$$

It then follows that,

$$
-\frac{2}{\sigma_{x}} \frac{d \sigma_{x}}{d \tau} \approx 2 \sqrt{a} \sigma_{0},-\frac{2}{\sigma_{x}} \frac{d \mu_{x}}{d \tau} \approx-8 \sqrt{a} \sigma_{0} \exp \left(-\sqrt{a} \sigma_{0} \tau\right)
$$

and,

$$
\begin{array}{r}
-\frac{1}{\sigma_{x}} \frac{d^{2} \mu_{x}(\tau)}{d \tau^{2}}+\frac{4}{\sigma_{x}^{2}} \frac{d \mu_{x}(\tau)}{d \tau} \frac{d \sigma_{x}(\tau)}{d \tau} \approx-8 a \sigma_{0}^{2} \exp \left(-\sqrt{a} \sigma_{0} \tau\right), \\
-\frac{1}{\sigma_{x}} \frac{d^{2} \sigma_{x}(\tau)}{d \tau^{2}}+\frac{1}{\sigma_{x}^{2}}\left(\frac{d \sigma_{x}(\tau)}{d \tau}\right)^{2}-\frac{1}{2 \sigma_{x}^{2}}\left(\frac{d \mu_{x}(\tau)}{d \tau}\right)^{2} \approx-8 a \sigma_{0}^{2} \exp \left(-2 \sqrt{a} \sigma_{0} \tau\right) .
\end{array}
$$

Therefore, setting $\Lambda_{3 D} \stackrel{\text { def }}{=} \lambda_{3 D} \sigma_{0}$ with $\lambda_{3 D}=\lambda_{+}^{\prime}$, the JLC equation for $J^{1}$ becomes,

$$
\frac{d^{2} J^{1}}{d \tau^{2}}+2 \Lambda_{3 D} \frac{d J^{1}}{d \tau}-8 \Lambda_{3 D} \exp \left(-\Lambda_{3 D} \tau\right) \frac{d J^{2}}{d \tau}-8 \Lambda_{3 D}^{2} \exp \left(-2 \Lambda_{3 D} \tau\right) J^{1}-8 \Lambda_{3 D}^{2} \exp \left(-\Lambda_{3 D} \tau\right) J^{2}=0 .
$$

For $J^{2}$ we get,

$$
\frac{D^{2} J^{2}}{D \tau^{2}}-\frac{1}{2 \sigma_{x}^{2}}\left[J^{2}\left(\frac{d \mu_{x}}{d \tau}\right)^{2}-J^{1} \frac{d \mu_{x}}{d \tau} \frac{d \sigma_{x}}{d \tau}\right]=0 .
$$

where we used the identity $R_{121}^{2}=-\frac{1}{2 \sigma_{x}^{2}}$. Notice that,

$$
\begin{aligned}
\frac{D^{2} J^{2}}{D \tau^{2}} & =\frac{d^{2} J^{2}}{d \tau^{2}}+2\left(\Gamma_{11}^{2} \frac{d J^{1}}{d \tau} \frac{d \theta^{1}}{d \tau}+\Gamma_{22}^{2} \frac{d J^{2}}{d \tau} \frac{d \theta^{2}}{d \tau}\right)+\left(\Gamma_{11}^{2} J^{1} \frac{d^{2} \theta^{1}}{d \tau^{2}}+\Gamma_{22}^{2} J^{2} \frac{d^{2} \theta^{2}}{d \tau^{2}}\right)+ \\
& +\left[\partial_{2} \Gamma_{11}^{2} \frac{d \theta^{1}}{d \tau} \frac{d \theta^{2}}{d \tau} J^{1}+\partial_{2} \Gamma_{22}^{2}\left(\frac{d \theta^{2}}{d \tau}\right)^{2} J^{2}\right]+ \\
& +\left[\Gamma_{11}^{2} \Gamma_{12}^{1} \frac{d \theta^{1}}{d \tau} \frac{d \theta^{2}}{d \tau} J^{1}+\Gamma_{11}^{2} \Gamma_{21}^{1}\left(\frac{d \theta^{1}}{d \tau}\right)^{2} J^{2}+\Gamma_{22}^{2} \Gamma_{11}^{2} \frac{d \theta^{1}}{d \tau} \frac{d \theta^{2}}{d \tau} J^{1}+\left(\Gamma_{22}^{2}\right)^{2}\left(\frac{d \theta^{2}}{d \tau}\right)^{2} J^{2}\right] .
\end{aligned}
$$


From (B16) and (B17), the JLC equation for $J^{2}$ becomes after some algebra,

$$
\begin{aligned}
0 & =\frac{d^{2} J^{2}}{d \tau^{2}}+2\left(\Gamma_{11}^{2} \frac{d J^{1}}{d \tau} \frac{d \theta^{1}}{d \tau}+\Gamma_{22}^{2} \frac{d J^{2}}{d \tau} \frac{d \theta^{2}}{d \tau}\right)+ \\
& +J^{1}\left[\Gamma_{11}^{2} \frac{d^{2} \theta^{1}}{d \tau^{2}}+\partial_{2} \Gamma_{11}^{2} \frac{d \theta^{1}}{d \tau} \frac{d \theta^{2}}{d \tau}+\Gamma_{11}^{2} \Gamma_{12}^{1} \frac{d \theta^{1}}{d \tau} \frac{d \theta^{2}}{d \tau}+\Gamma_{22}^{2} \Gamma_{11}^{2} \frac{d \theta^{1}}{d \tau} \frac{d \theta^{2}}{d \tau}-R_{121}^{2} \frac{d \theta^{1}}{d \tau} \frac{d \theta^{2}}{d \tau}\right]+ \\
& +J^{2}\left[\Gamma_{22}^{2} \frac{d^{2} \theta^{2}}{d \tau^{2}}+\partial_{2} \Gamma_{22}^{2}\left(\frac{d \theta^{2}}{d \tau}\right)^{2}+\Gamma_{11}^{2} \Gamma_{21}^{1}\left(\frac{d \theta^{1}}{d \tau}\right)^{2}+\left(\Gamma_{22}^{2}\right)^{2}\left(\frac{d \theta^{2}}{d \tau}\right)^{2}+R_{121}^{2}\left(\frac{d \theta^{1}}{d \tau}\right)^{2}\right] .
\end{aligned}
$$

Recalling that $\theta^{1}=\mu_{x}$ and $\theta^{2}=\sigma_{x}$, we get

$$
\begin{aligned}
0 & =\frac{d^{2} J^{2}}{d \tau^{2}}+2 \Gamma_{11}^{2} \frac{d \mu_{x}}{d \tau} \frac{d J^{1}}{d \tau}+2 \Gamma_{22}^{2} \frac{d \sigma_{x}}{d \tau} \frac{d J^{2}}{d \tau}+ \\
& +J^{1}\left[\Gamma_{11}^{2} \frac{d^{2} \mu_{x}}{d \tau^{2}}+\left(\partial_{2} \Gamma_{11}^{2}+\Gamma_{11}^{2} \Gamma_{12}^{1}+\Gamma_{22}^{2} \Gamma_{11}^{2}-R_{121}^{2}\right) \frac{d \mu_{x}}{d \tau} \frac{d \sigma_{x}}{d \tau}\right]+ \\
& +J^{2}\left[\Gamma_{22}^{2} \frac{d^{2} \sigma_{x}}{d \tau^{2}}+\left(\partial_{2} \Gamma_{22}^{2}+\left(\Gamma_{22}^{2}\right)^{2}\right)\left(\frac{d \sigma_{x}}{d \tau}\right)^{2}+\left(\Gamma_{11}^{2} \Gamma_{21}^{1}+R_{121}^{2}\right)\left(\frac{d \mu_{x}}{d \tau}\right)^{2}\right] .
\end{aligned}
$$

Noting that the following relations hold,

$$
\partial_{2} \Gamma_{11}^{2}+\Gamma_{11}^{2} \Gamma_{12}^{1}+\Gamma_{22}^{2} \Gamma_{11}^{2}-R_{121}^{2}=-\frac{1}{\sigma_{x}^{2}}, \partial_{2} \Gamma_{22}^{2}+\left(\Gamma_{22}^{2}\right)^{2}=\frac{2}{\sigma_{x}^{2}} \text { and, } \Gamma_{11}^{2} \Gamma_{21}^{1}+R_{121}^{2}=-\frac{1}{\sigma_{x}^{2}},
$$

Eq. (B19) becomes,

$$
\begin{aligned}
0 & =\frac{d^{2} J^{2}}{d \tau^{2}}+\left(\frac{1}{\sigma_{x}} \frac{d \mu_{x}}{d \tau}\right) \frac{d J^{1}}{d \tau}+\left(-\frac{2}{\sigma_{x}} \frac{d \sigma_{x}}{d \tau}\right) \frac{d J^{2}}{d \tau}+ \\
& +J^{1}\left[\frac{1}{2 \sigma_{x}} \frac{d^{2} \mu_{x}}{d \tau^{2}}-\frac{1}{\sigma_{x}^{2}} \frac{d \mu_{x}}{d \tau} \frac{d \sigma_{x}}{d \tau}\right]+ \\
& +J^{2}\left[-\frac{1}{\sigma_{x}} \frac{d^{2} \sigma_{x}}{d \tau^{2}}+\frac{2}{\sigma_{x}^{2}}\left(\frac{d \sigma_{x}}{d \tau}\right)^{2}-\frac{1}{\sigma_{x}^{2}}\left(\frac{d \mu_{x}}{d \tau}\right)^{2}\right]
\end{aligned}
$$

From (B12) we get,

$$
\frac{1}{\sigma_{x}} \frac{d \mu_{x}}{d \tau} \approx 4 \sqrt{a} \sigma_{0} \exp \left(-\sqrt{a} \sigma_{0} \tau\right),-\frac{2}{\sigma_{x}} \frac{d \sigma_{x}}{d \tau} \approx 2 \sqrt{a} \sigma_{0}
$$

and,

$$
\begin{gathered}
\frac{1}{2 \sigma_{x}} \frac{d^{2} \mu_{x}(\tau)}{d \tau^{2}}-\frac{1}{\sigma_{x}^{2}} \frac{d \mu_{x}(\tau)}{d \tau} \frac{d \sigma_{x}(\tau)}{d \tau} \approx 0, \\
-\frac{1}{\sigma_{x}} \frac{d^{2} \sigma_{x}(\tau)}{d \tau^{2}}+\frac{2}{\sigma_{x}^{2}}\left(\frac{d \sigma_{x}(\tau)}{d \tau}\right)^{2}-\frac{1}{\sigma_{x}^{2}}\left(\frac{d \mu_{x}(\tau)}{d \tau}\right)^{2} \approx a \sigma_{0}^{2} .
\end{gathered}
$$

Substituting (B22) and (B23) into (B21), we obtain

$$
\frac{d^{2} J^{2}}{d \tau^{2}}+4 \Lambda_{3 D} \exp \left(-\Lambda_{3 D} \tau\right) \frac{d J^{1}}{d \tau}+2 \Lambda_{3 D} \frac{d J^{2}}{d \tau}+\Lambda_{3 D}^{2} J^{2}=0
$$


Finally, the JLC equation for $J^{3}$ is given by,

$$
\frac{D^{2} J^{3}}{D \tau^{2}}=0
$$

Notice that,

$$
\frac{D^{2} J^{3}}{D \tau^{2}}=\frac{d^{2} J^{3}}{d \tau^{2}}+\left(2 \Gamma_{33}^{3} \frac{d \theta^{3}}{d \tau}\right) \frac{d J^{3}}{d \tau}+J^{3}\left[\Gamma_{33}^{3} \frac{d^{2} \theta^{3}}{d \tau^{2}}+\left(\partial_{3} \Gamma_{33}^{3}+\left(\Gamma_{33}^{3}\right)^{2}\right)\left(\frac{d \theta^{3}}{d \tau}\right)^{2}\right]
$$

Therefore, the JLC equation for $J^{3}$ becomes,

$$
\frac{d^{2} J^{3}}{d \tau^{2}}+\left(-\frac{2}{\sigma_{y}} \frac{d \sigma_{y}}{d \tau}\right) \frac{d J^{3}}{d \tau}+J^{3}\left[-\frac{1}{\sigma_{y}} \frac{d^{2} \sigma_{y}}{d \tau^{2}}+\frac{2}{\sigma_{y}^{2}}\left(\frac{d \sigma_{y}}{d \tau}\right)^{2}\right]=0
$$

having recalled that,

$$
\Gamma_{33}^{3}=-\frac{1}{\sigma_{y}} \text { and, } \partial_{3} \Gamma_{33}^{3}+\left(\Gamma_{33}^{3}\right)^{2}=\frac{2}{\sigma_{y}^{2}}
$$

Observing that,

$$
\begin{gathered}
\sigma_{y}(\tau)=\sigma_{0}^{\prime} \exp \left(-\lambda_{f} \tau\right), \frac{d \sigma_{y}(\tau)}{d \tau}=-\sigma_{0}^{\prime} \lambda_{f} e^{-\tau \lambda_{f}},\left(\frac{d \sigma_{y}(\tau)}{d \tau}\right)^{2}=\sigma_{0}^{\prime 2} \lambda_{f}^{2} e^{-2 \tau \lambda_{f}}, \\
\frac{d^{2} \sigma_{y}(\tau)}{d \tau^{2}}=\sigma_{0}^{\prime} \lambda_{f}^{2} e^{-\tau \lambda_{f}},-\frac{2}{\sigma_{y}} \frac{d \sigma_{y}(\tau)}{d \tau}=2 \lambda_{f},-\frac{1}{\sigma_{y}} \frac{d^{2} \sigma_{y}(\tau)}{d \tau^{2}}+\frac{2}{\sigma_{y}^{2}}\left(\frac{d \sigma_{y}(\tau)}{d \tau}\right)^{2}=\lambda_{f}^{2},
\end{gathered}
$$

the JLC equation for $J^{3}$ becomes,

$$
\frac{d^{2} J^{3}}{d \tau^{2}}+2 \lambda_{f} \frac{d J^{3}}{d \tau}+\lambda_{f}^{2} J^{3}=0
$$

Following the same line of reasoning, we are capable of computing (and integrating) also the JLC equations for the $2 D$ statistical model.

[1] R. Landauer, " A simple measure of complexity", Nature 336, 306 (1988).

[2] M. Gell-Mann, "What is Complexity?", Complexity 1, 1 (1995).

[3] D. P. Feldman and J. P. Crutchfield, "Measures of statistical complexity: Why?", Phys. Lett. A238, 244 (1998).

[4] G. Benenti and G. Casati, "How complex is quantum motion?", Phys. Rev E79, 025201 (2009).

[5] M. Ohya, "Complexities and Their Applications to Characterization of Chaos", Int. J. Theor. Phys. 37, 495 (1998).

[6] S. Wolfram, "Universality and Complexity in Cellular Automata", Physica D10, 1 (1984).

[7] S. Wolfram, "Origin of Randomness in Physical Systems", Phys. Rev. Lett. 55, 449 (1985).

[8] M. Rasetti, "Uncertainty, Predictability and Decidability in Chaotic Dynamical Systems", Chaos, Solitons \& Fractals 5, 133 (1995).

[9] B. A. Huberman and T. Hogg, " Complexity and Adaption", Physica D22, 376 (1986).

[10] P. Grassberger, "Toward a Quantitative Theory of Self-Generated Complexity", Int. J. Theor. Phys. 25, 907 (1986).

[11] J. P. Crutchfield and K. Young, "Inferring Statistical Complexity", Phys. Rev. Lett. 63, 105 (1989).

[12] A. N. Kolmogorov, "Three approaches to the quantitative definition of information", Probl. Inf. Transm. (USSR) 1, 4 (1965); "Logical basis for information theory and probability theory", IEEE Trans. Inf. Theory, IT14, 662 (1968).

[13] Y. Pesin, "Characteristic Lyapunov exponents and smooth ergodic theory", English transl., Russian Math. Surveys 32, 55 (1977).

[14] A. A. Brudno, "Entropy and the complexity of trajectories of a dynamical system", Trans. Moscow Math. Soc. 2, 127 (1983).

[15] F. Blume, "On the relation between entropy and the average complexity of trajectories in dynamical systems", Comp. Complex. 9, 146 (2000).

[16] P. Szepfalusy, "Characterization of Chaos and Complexity by Properties of Dynamical Entropies", Physica Scripta T25, 226 (1989). 
[17] A. Wehrl, "General properties of entropy", Rev. Mod. Phys. 50, 221 (1978).

[18] C. H. Bennett, "How to Define Complexity in Physics, and Why", in "Complexity, Entropy and the Physics of Information", SFI Studies in the Sciences of Complexity, vol.VIII, Ed. W. H. Zurek, Addison-Wesley (1990).

[19] S. Lloyd and H. Pagels, "Complexity as Thermodynamic Depth", Annals of Physics 188, 186 (1988).

[20] C. M. Papadimitriou, "Computational Complexity", Addison-Wesley, Massachusetts (1994).

[21] J. Rissanen, "Stochastic complexity and modeling", Ann. Stat. 14, 1080 (1986).

[22] L. A. Caron et al., "Quantum chaos at finite temperature", Phys. Lett. A288, 145 (2001).

[23] L. A. Caron et al., "Is Quantum Chaos Weaker Than Classical Chaos?", Phys. Lett. A322, 60 (2004).

[24] H. Kroger et al., "Quantum Chaos Versus Classical Chaos: Why is Quantum Chaos Weaker?", arXiv:quant-ph/0603084 (2006).

[25] A. Peres, "Quantum Theory: Concepts and Methods", Fundamental Theories of Physics.

[26] C. Cafaro and S. Mancini, "Quantifying The Complexity Of Geodesic Paths On Curved Statistical Manifolds Through Information Geometric Entropies and Jacobi Fields", Physica D240, 607 (2011).

[27] A. Caticha, "Entropic Dynamics", in Bayesian Inference and Maximum Entropy Methods in Science and Engineering, ed. by R.L. Fry, AIP Conf. Proc. 617, 302 (2002).

[28] S. Amari and H. Nagaoka, "Methods of Information Geometry", American Mathematical Society, Oxford University Press (2000).

[29] A. Giffin, "Maximum Entropy: The Universal Method for Inference", Ph. D. Thesis, SUNY at Albany, NY-USA (2008).

[30] A. Caticha and R. Preuss, "Maximum entropy and Bayesian data analysis: Entropic prior distributions", Phys. Rev. E70, 046127 (2004).

[31] J. E. Shore and R. W. Johnson, IEEE Trans. Inf. Theory IT-26, 26 (1980); IEEE Trans. Inf. Theory IT-27, 26 (1981).

[32] J. Skilling, "The Axioms of Maximum Entropy", Maximum-Entropy and Bayesian Methods in Science and Engineering, G. J. Erickson and C. R. Smith (eds.) (Kluwer, Dordrecht, 1988).

[33] A. Caticha and A. Giffin, "Updating Probabilities", in Bayesian Inference and Maximum Entropy Methods in Science and Engineering, ed. by Ali Mohammad-Djafari, AIP Conf. Proc. 872, 31 (2006).

[34] C. Cafaro, "The Information Geometry of Chaos", Ph. D. Thesis, SUNY at Albany, NY-USA (2008).

[35] C. Cafaro, "Works on an information geometrodynamical approach to chaos", Chaos, Solitons \& Fractals 41, 886 (2009).

[36] L. Casetti et al., "Riemannian theory of Hamiltonian chaos and Lyapunov exponents", Phys. Rev. E54, 5969 (1996).

[37] M. Di Bari and P. Cipriani, " Geometry and Chaos on Riemann and Finsler Manifolds", Planet. Space Sci. 46, 1543 (1998).

[38] C. G. J. Jacobi, "Vorlesungen uber Dynamik", Reimer, Berlin (1866).

[39] L. D. Landau and E. M. Lifshitz, "The Classical Theory of Fields", Pergamon, London (1962).

[40] C. Cafaro, "Information-geometric indicators of chaos in Gaussian models on statistical manifolds of negative Ricci curvature", Int. J. Theor. Phys. 47, 2924 (2008).

[41] C. Cafaro et al., "Reexamination of An Information Geometric Construction of Entropic Indicators of Complexity", Appl. Math. Comput. 217, 2944 (2010).

[42] F. De Felice and J. S. Clarke, "Relativity on Curved Manifolds", Cambridge University Press (1990).

[43] H. C. Ohanian and R. Ruffini, "Gravitation and Spacetime", W.W. Norton \& Company (1994).

[44] C. Chicone and B. Mashhoon, "The generalized Jacobi equation", Class. Quantum Grav. 19, 4231 (2002).

[45] G. P. Berman and G. M. Zaslavsky, "Condition of stochasticity in quantum nonlinear systems", Physica A91, 450 (1978).

[46] G. Casati and B. Chirikov, "Quantum chaos: unexpected complexity", Physica D86, 220 (1995).

[47] A. Caticha and C. Cafaro, "From information geometry to Newtonian dynamics", in Bayesian Inference and Maximum Entropy Methods in Science and Engineering, AIP Conf. Proc. 954, 165 (2007).

[48] P. Ishwar and P. Moulin, " On the existence and characterization of the maxent distribution under general moment inequality constraints", IEEE Trans. Inf. Theory 51, 3322 (2005).

[49] L. Peng et al., "The geometric structures and instability of entropic dynamical models", Adv. Math. 227, 459 (2011). 